Sciences

Vol. 06, No. 03, pp. 64-82, September 2013

\title{
CALCULATION OF A THREE DIMENSIONAL COORDINATES OF A MANIPULATOR END EFFECTOR IN A COMPOUND FRAMES
}

\author{
Emad K. Hussein \\ Foundation of Technical Education $\backslash$ Al-Mussaib Technical College \\ E-mail: emad_kamil72@yahoo.com \\ (Received:1/3/2012; Accepted:5/11/2012)
}

\begin{abstract}
A theoretical and experimental study of measurement of three dimensional Cartesian coordinates of the end effecter of a robotic manipulator with three different frames each one known relative to the other was carried out in this research.

The aim of this work is, first, to calculate three dimensional Cartesian coordinates ${ }^{A} P_{X},{ }^{A} P_{Y},{ }^{A} P_{Z}$ of an end effecter tip which lies in a specific frame $\{\mathrm{C}\}$ with respect to another frame which represents the fixed base frame $\{A\}$ passing through an intermediate frame $\{B\}$ by using Matrix Compound Transformation Method i.e. theoretical approach, then followed by measuring the same coordinates by another two traditional procedures, they are:

○ Self Coordinates Feeding.

- Manual Alignment.
\end{abstract}

And comparing the final obtained results (theoretical and experimental) with the standard one. The second step is checking repeatability for the above mentioned methods, by measuring the final tip coordinates for 18 times for each case.

The final result shows that the Matrix Compound Transformation Method gives an accurate repeatability comparing with the others.

Keywords:-End effectors, Position description, Compound frames, Repeatability.

\section{1- INTRODUCTION}

Robotic manipulation, by definition, implies that part and tools will be moved around in space by some sort of mechanism. This naturally leads to the need of representing positions and accelerations of the parts, tools and of the mechanism itself. To define and 
manipulate mathematical quantities which represent position and orientation we must define coordinate systems and develop contentions for representation ${ }^{(1)}$.

The researcher adopt the philosophy that somewhere there is a universe coordinate system to which everything we discuss can be referenced. The researcher will describe all positions and orientations with respect to the universe coordinate system or with respect to the Cartesian coordinate system which could be defined relative to the universe system.

\section{2- DESCRIPTIONS: POSITIONS, ORIENTATIONS, AND FRAMES}

A description is used to specify attributes of various objects with which a manipulation system deals. These objects are parts, tools or perhaps the manipulator itself ${ }^{(1,}$ 2).

\section{1) DESCRIPTION OF A POSITION}

Once a coordinate system is established we can locate any point in the universe with a $3 * 1$ position vector. Because we will often define many coordinate systems in addition to the universe coordinate system, vectors must be tagged with information identifying which coordinate system they are defined within. In this research vectors are written with a leading superscript indicating the coordinate system to which they are referenced, for example ${ }^{A} P$. This means that the components of ${ }^{A} P$ have numerical values which indicate distance along the axes of $\{\mathrm{A}\}$. Each of these distances along an axis can be thought of as the result of projecting the vector onto the corresponding axis.

Figure (1) pictorially represents a coordinate system, $\{A\}$, with three mutually orthogonal unit vectors with solid head. A point ${ }^{A} P$ is represented with a vector and can be equivalently being thought of as a position in space, or simply as an ordered set of three numbers. Individual elements of a vector are given subscripts $x, y$, and $z:[1,2,3]$

$$
\text { (1) }{ }^{A} P=\left[\begin{array}{c}
p_{x} \\
p_{y} \\
p_{z}
\end{array}\right]
$$

\section{2) DESCRIPTION OF AN ORIENTATION}

Often it is necessary not only to represent a point in space but also to describe the orientation of a body in space. For example if vector ${ }^{A} P$ in figure (2) locates the point 
directly between the fingertips of a manipulator $\mathrm{s}$ hand, the complete location of the hand is still not specified until its orientation is also given. In order to describe the orientation of a body we will attach a coordinate system to the body and then give a description of this coordinate system relative to the reference system. In figure (2) coordinate system $\{B\}$ has been attached to the body in a known way. A description of $\{B\}$ relative to $\{A\}$ now suffices to give the orientation of the body. Thus, positions of points are described with vectors and orientations of bodies are described with an attached coordinate system. One way to describe the body-attached coordinate system, $\{B\}$, is to write the unit vectors of its three principal axes in terms of the coordinate system $\{\mathrm{A}\}$.

We denote the unit vectors giving the principal directions of coordinate system $\{\mathrm{B}\}$ as $X_{B}, Y_{B}$, and $Z_{B}$. When written in terms of coordinate system $\{\mathrm{A}\}$ they are called ${ }^{A} X_{B}$, ${ }^{A} Y_{B}$ and ${ }^{A} Z_{B}$. It will be convenient if we stack these three unit vectors together as the columns of a $3 * 3$ matrix, in the $\operatorname{order}^{A} X_{B},{ }^{A} Y_{B}$ and ${ }^{A} Z_{B}$. We will call this matrix a rotation matrix, and because this particular rotation matrix describes $\{B\}$ relative to $\{A\}$, we name it with the notation ${ }_{B}^{A} R^{(1,2)}$.

$$
{ }_{B}^{A} R=\left[\begin{array}{lll}
{ }^{A} X_{B} & { }^{A} Y_{B} & { }^{A} Z_{B}
\end{array}\right]=\left[\begin{array}{lll}
r_{11} & r_{12} & r_{13} \\
r_{21} & r_{22} & r_{23} \\
r_{31} & r_{32} & r_{33}
\end{array}\right]
$$

We can give expression for the scalars ${ }^{r_{i j}}$ in equation (2) by noting that the components of any vectors are simply the projection of that vector onto the unit direction of its reference frame. Hence each component of ${ }_{B}^{A} R$ in equation (2) can be written as the dot product of a pair of unit vectors as $(2,4)$ :

$$
{ }_{B}^{A} R=\left[\begin{array}{lll}
{ }^{A} X_{B} & { }^{A} Y_{B} & { }^{A} Z_{B}
\end{array}\right]=\left[\begin{array}{ccc}
X_{B} \cdot X_{A} & Y_{B} \cdot X_{A} & Z_{B} \cdot X_{A} \\
X_{B} \cdot Y_{A} & Y_{B} \cdot Y_{A} & Z_{B} \cdot Y_{A} \\
X_{B} \cdot Z_{A} & Y_{B} \cdot Z_{A} & Z_{B} \cdot Z_{A}
\end{array}\right]
$$

Since the dot product of two unit vectors yields the cosine of the angle between them it is clear why the components of rotation matrices are often referred to as direction cosines. Further inspection of equation (3) shows that the rows of the matrix are the unit vector of $\{\mathrm{A}\}$ expressed in $\{\mathrm{B}\}$; that is, 
${ }_{B}^{A} R=\left[\begin{array}{lll}{ }^{A} X_{B} & { }^{A} Y_{B} & { }^{A} Z_{B}\end{array}\right]=\left[\begin{array}{c}{ }^{B} X_{A}^{T} \\ { }^{B} Y_{A}^{T} \\ { }^{B} Z_{A}^{T}\end{array}\right]$

Hence ${ }_{A}^{B} R$ the description of frame $\{\mathrm{A}\}$ relative to $\{\mathrm{B}\}$ is given by the transpose of equation (3); that is,

$$
{ }_{A}^{B} R={ }_{B}^{A} R^{T}
$$

This suggests that the inverse of a rotation matrix is equal to its transpose a fact which can be verified as $(12,15)$

${ }_{B}^{A} R^{T}{ }_{B}^{A} R=\left[\begin{array}{c}{ }^{A} X_{B}^{T} \\ { }^{A} Y_{B}^{T} \\ { }^{A} Z_{B}^{T}\end{array}\right]\left[\begin{array}{lll}{ }^{A} X_{B} & { }^{A} Y_{B} & { }^{A} Z_{B}\end{array}\right]=I_{3}$

Where $I_{3}$ is the $3 * 3$ identity matrix. Hence,

${ }_{B}^{A} R={ }_{A}^{B} R^{-1}={ }_{A}^{B} R^{T}$

\section{3) DESCRIPTION OF A FRAME}

The information needed to completely specify the whereabouts of the manipulator hand in figure (2) is a position and orientation. The point on the body whose position we describe could be chosen arbitrarily, however for convenience the point whose position we will describe is chosen as the origin of the body attached frame. The situation of a position and an orientation pair arises so often in robotics that we define an entity called a frame, which is a set of four vectors giving position and orientation information. For example in figure (2) one vector locates the fingertip position and three more describe its orientation. Equivalently the description of a frame can be thought of as a position vector and rotation matrix. Note that a frame is a coordinate system where in addition to the orientation we give a position vector which locates its origin relative to some other embedding frame. For example frame $\{\mathrm{B}\}$ is described by ${ }_{B}^{A} R$ and ${ }^{A} P_{B O R G}$ where ${ }^{A} P_{B O R G}$ is the vector which locates the origin of the frame $\{B\}^{(5,6,7,8)}$.

$$
\{B\}=\left\{{ }_{B}^{A} R, \quad{ }^{A} P_{B O R G}\right\}
$$

In figure (3) there are three frames that are shown along with the inverse coordinate system. Frame $\{A\}$ and $\{B\}$ are known relative to the universe coordinate system and frame $\{C\}$ is known relative to frame $\{A\}$. In figure (3) we introduce a graphical representation of 
frames which is convenient in visualizing frames. A frame is depicted by three arrows representing unit vectors defining the principal axes of the frame. An arrow representing a vector is drawn from one origin to another. This vector represents the position of the origin at the head of the arrow in terms of the frame at the tail of the arrow. The direction of this locating arrow tells us, for example in figure (3) that $\{C\}$ is known relative to $\{A\}$ and not vice versa.

\section{MAPPINGS}

\section{1) MAPPINGS INVOLVING TRANSLATED FRAMES}

In figure (4) we have a position defined by the vector ${ }^{B} P$. The researcher wishes to express this point in space in terms of frame $\{A\}$, when $\{A\}$ has the same orientation as $\{B\}$. In this case, $\{\mathrm{B}\}$ differs from $\{\mathrm{A}\}$ only by a translation which is given by ${ }^{A} P_{B O R G}$, a vector which locates the origin of $\{\mathrm{B}\}$ relative to $\{\mathrm{A}\}$. Because both vectors are defined relative to frames of the same orientation, we calculate the description of point $\mathrm{P}$ relative to $\{\mathrm{A}\},{ }^{A} P$ by vector edition:

$$
{ }^{A} P={ }^{B} P+{ }^{A} P_{B O R G}
$$

We say that the vector ${ }^{A} P_{B O R G}$ defines this mapping, since all the information needed to perform the change in description is contained in ${ }^{A} P_{B O R G}{ }^{(1,2,3)}$.

\section{2) MAPPINGS INVOLVING ROTATED FRAMES}

By our definition, the columns of a rotation matrix all have unit magnitude, and further, these unit vectors are orthogonal. As we saw in the previous articles, a consequence of this is that

${ }_{B}^{A} R={ }_{A}^{B} R^{-1}={ }_{A}^{B} R^{T}$

Therefore, since the columns of ${ }_{B}^{A} R$ are the unit vectors of $\{\mathrm{B}\}$ written in $\{\mathrm{A}\}$, then the rows of ${ }_{B}^{A} R$ are the unit vectors of $\{\mathrm{A}\}$ written in $\{\mathrm{B}\}$. So a rotation matrix can be interpreted as a set of three column vectors are as a set of three row vectors as follows:

$$
{ }_{B}^{A} R=\left[\begin{array}{lll}
{ }^{A} X_{B} & { }^{A} Y_{B} & { }^{A} Z_{B}
\end{array}\right]=\left[\begin{array}{c}
{ }^{B} X_{A}^{T} \\
{ }^{B} Y_{A}^{T} \\
{ }^{B} Z_{A}^{T}
\end{array}\right]
$$


As in figure (5), the situation will arise often where we know the definition of a vector with respect to some frame, $\{B\}$, and we would like to know its definition with respect to another frame $\{\mathrm{A}\}$, where the origins of the two frames are coincident. This computation is possible when a description of the orientation of $\{B\}$ is known relative to $\{A\}$. This orientation is given by the rotation matrix ${ }_{B}^{A} R$, whose columns are the unit vector of $\{\mathrm{B}\}$ written in $\{\mathrm{A}\}$. In order to calculate ${ }^{A} P$, we note that the components of any vector are simply the projections of that vector onto the unit directions of its frame. The projection is calculated with the vector dot product. Thus we see that the components of ${ }^{A} P$ may be calculated as: $\left.{ }^{9}, 10,11\right)$

$$
\begin{aligned}
& { }^{A} p_{x}={ }^{B} X_{A} \cdot{ }^{B} P \\
& { }^{A} p_{y}={ }^{B} Y_{A} \cdot{ }^{B} P \\
& { }^{A} p_{z}={ }^{B} Z_{A} \cdot{ }^{B} P
\end{aligned}
$$

In order to express equation (11) in terms of a rotation matrix multiplication, we note from equation (10) that the rows of ${ }_{B}^{A} R$ are ${ }^{B} X_{A},{ }^{B} Y_{A}$ and ${ }^{B} Z_{A}$ so equation (11) may be written compactly using a rotation matrix as:

$$
{ }^{A} P={ }_{B}^{A} R^{B} P
$$

\section{3) MAPPINGS INVOLVING GENERAL FRAMES}

Very often we know the description of a vector with respect to some frame, $\{B\}$, and we would like to know its description with respect to another frame, $\{\mathrm{A}\}$. We now consider the general case of mapping. Here the origin of frame $\{B\}$ is not coincident with that of frame $\{\mathrm{A}\}$ but has a general vector offset. The vector that locates $\{\mathrm{B}\}$ 's origin is called ${ }^{A} P_{B O R G}$. Also $\{\mathrm{B}\}$ is rotated with respect to $\{\mathrm{A}\}$ as described by ${ }_{B}^{A} R$. Given ${ }^{B} P$, we wish to compute ${ }^{A} P$ as shown in figure (6). We can first change ${ }^{B} P$ to its description relative to an intermediate frame which has the same orientation as $\{\mathrm{A}\}$, but whose origin is coincident with the origin of $\{\mathrm{B}\}$. This is done by premultiplying by ${ }_{B}^{A} R$. We then account for the translation between origins by simple vector addition, yielding:

$$
{ }^{A} P={ }_{B}^{A} R{ }^{B} P+{ }^{A} P_{B O R G}
$$

Equation (13) describes a general transformation mapping of a vector from its description in one frame to a description in a second frame. We would like to think of a 
mapping from one frame to another as an operator in matrix form. This aids in writing compact equations as well as being conceptually clearer than equation (13). We define a $4 * 4$

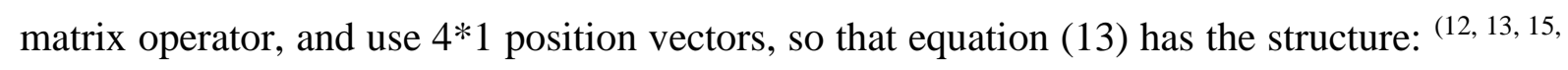
16)

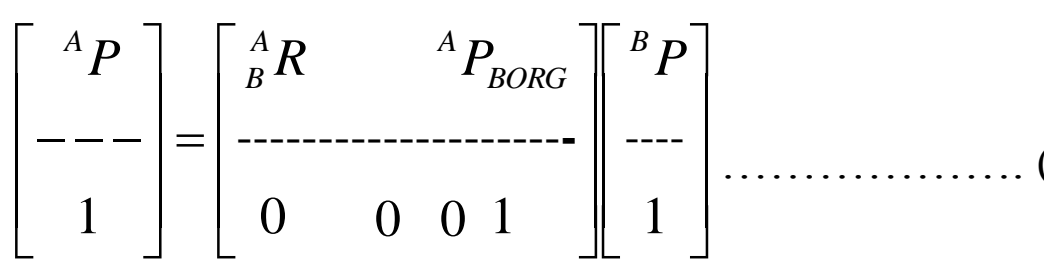

We adopt the convention that a position vector is $3 * 1$ or $4 * 1$ depending on whether it appears multiplied by a $3 * 3$ matrix or by a $4 * 4$ matrix. It is readily seen that equation (14) implements

$$
\begin{aligned}
& { }^{A} P={ }_{B}^{A} R^{B} P+{ }^{A} P_{B O R G} \\
& 1=1
\end{aligned}
$$

The $4 * 4$ matrix in equation (14) is called a homogeneous transform. For our purpose it can be regarded purely as a construction used to cast the rotation and translation of the general transform into a single matrix form.

\section{TRANSFORMATION}

\section{1) COMPOUND TRANSFORMATION}

In figure (7), we have ${ }^{C} P$ and wish to find ${ }^{A} P$. Frame $\{C\}$ is known relative to frame $\{\mathrm{B}\}$, and frame $\{\mathrm{B}\}$ is known relative to frame $\{\mathrm{A}\}$. We can transform ${ }^{C} P$ into ${ }^{B} P$ as ${ }^{B} P={ }_{C}^{B} T^{C} P$

And then transform ${ }^{B} P$ into ${ }^{A} P$ as

${ }^{A} P={ }_{B}^{A} T^{B} P$

Combining equation (16) and equation (17) we get the following: [15]

${ }^{A} P={ }_{B}^{A} T_{C}^{B} T^{C} P$

From which we could define:

${ }_{C}^{A} T={ }_{B}^{A} T_{C}^{B} T$ 
Again note that familiarity with the sub and superscript notation makes these manipulations simple. In terms of the known descriptions of $\{B\}$ and $\{C\}$, we can give the expression for ${ }_{C}^{A} T$ as:

$$
{ }_{C}^{A} T=\left[\begin{array}{cccc}
{ }_{B}^{A} R_{C}^{B} R & & { }_{B}^{A} R^{B} P_{C O R G}+{ }^{A} P_{B O R G} \\
--------------- & 0 & 0
\end{array}\right] \ldots
$$

\section{2) REPRESENTATION OF ORIENTATION}

\subsection{1) X-Y-Z FIXED ANGLES}

One method of describing the orientation of a frame $\{B\}$ is as follows: start with the frame coincident with a known reference frame $\{\mathrm{A}\}$. First rotate $\{\mathrm{B}\}$ about $X_{A}$ by an angle $\gamma$ then rotate about $Y_{A}$ by an angle $\beta$ and then rotate about $Z_{A}$ by an angle $\alpha$.

Each of the three rotations takes place about an axis in the fixed reference frame, $\{\mathrm{A}\}$. We will call this convention for specifying orientation $\mathrm{X}-\mathrm{Y}-\mathrm{Z}$ fixed angles. The word fixed refers to the fact that the rotations are specified about the fixed (i.e. non-moving) reference frame as shown in figure (8). Sometimes this convention is referred to as roll, pitch, and yaw angles.

The derivation of the equivalent rotation matrix, ${ }_{B}^{A} R_{X Y Z}(\gamma, \beta, \alpha)$ is straightforward because all rotations occur about axes of the reference frame

$$
\begin{aligned}
{ }_{B}^{A} R_{X Y Z}(\gamma, \beta, \alpha) & =R_{Z}(\alpha) \cdot R_{Y}(\beta) \cdot R_{X}(\gamma) \\
& =\left[\begin{array}{ccc}
c \alpha & -s \alpha & 0 \\
s \alpha & c \alpha & 0 \\
0 & 0 & 1
\end{array}\right]\left[\begin{array}{ccc}
c \beta & 0 & s \beta \\
0 & 1 & 0 \\
-s \beta & 0 & c \beta
\end{array}\right]\left[\begin{array}{ccc}
1 & 0 & 0 \\
0 & c \gamma & -s \gamma \\
0 & s \gamma & c \gamma
\end{array}\right]
\end{aligned}
$$

Where $\alpha \alpha$ is shorthand for $\cos (\alpha)$ and $\operatorname{s} \alpha$ for $\sin (\alpha)$, etc. It is extremely important to understand the order of rotations used in equation (21). Thinking in terms of rotations as operators, we have applied the rotation (from the right) of $R_{X}(\gamma)$, then $R_{Y}(\beta)$, and then $R_{Z}(\alpha)$. Multiplying equation (21) out, yielding:

$$
{ }_{B}^{A} R_{X Y Z}(\gamma, \beta, \alpha)=\left[\begin{array}{ccc}
c \alpha c \beta & c \alpha s \beta s \gamma-s \alpha c \gamma & c \alpha s \beta c \gamma+s \alpha s \gamma \\
s \alpha c \beta & s \alpha s \beta s \gamma+c \alpha c \gamma & s \alpha s \beta c \gamma-c \alpha s \gamma \\
-s \beta & c \beta s \gamma & c \beta c \gamma
\end{array}\right] \ldots \ldots
$$


Equation (22) is correct only for rotation performed in the order: about $X_{A}$ by $\gamma$, about $Y_{A}$ by $\beta$, about $Z_{A}$ by $\alpha$.

\section{REPEATABILITY}

Repeatability is a statistical term which provides a measure of the ability of the robot to return the end effecter repeatedly and consistently to a previous taught point. It can be taken as the length of the radius of the smallest circle that can be drawn to include all tool center points achieved ${ }^{(17)}$.

Repeatability means doing the same thing over and over within a given tolerance. Consider the case of a manipulator operating in a system of rectangular coordinates as that described in the previous articles ${ }^{(18)}$. The manipulator is supposed to move from the point of origin to the position $X=3 \mathrm{~m}, Y=4 \mathrm{~m}$, and $\mathrm{Z}=12 \mathrm{~m}$. Let the stated repeatability be $0.001 \mathrm{~m}$ and all axes be affected by the same maximum deviation. The length of the motion in a system of rectangular coordinates is given by $\left(X^{2}+Y^{2}+Z^{2}\right)$. In the ideal case this is $13 \mathrm{~m}$. Consider however an error in all three axis of $0.001 \mathrm{~m}$, the movement will be $13.002 \mathrm{~m}$ this means the resulting error will be $13.002-13=0.002 \mathrm{~m}$ rather than $0.001 \mathrm{~m}$, which means a difference between stated and actual error. See figures (10), (11) and (12).

\section{CALCULATIONS AND RESULTS}

If there are three frames $\{A\},\{B\}$, and $\{C\}$ as shown in figure (9) below with a specific parameters and it is required to find the three dimensional coordinates of the point $\mathrm{P}$ which lies in frame $\{C\}$ with respect to the reference frame $\{A\}$ passing through an intermediate frame $\{\mathrm{B}\}$, then we will follow the following procedure:

$$
\begin{aligned}
& { }^{A} P={ }_{B}^{A} T_{C}^{B} T^{C} P \\
& { }^{A} P={ }_{C}^{A} T^{C} P \\
& { }_{C}^{A} T=\left[\begin{array}{cc}
{ }_{B}^{A} R_{C}^{B} R & { }_{B}^{A} R^{B} P_{C O R G}+{ }^{A} P_{B O R G} \\
0 & 1
\end{array}\right] \\
& { }_{C, B}^{B, A} R_{r p y}(\gamma, \beta, \alpha)=\left[\begin{array}{ccc}
c \alpha c \beta & c \alpha s \beta s \gamma-s \alpha c \gamma & c \alpha s \beta c \gamma+s \alpha s \gamma \\
s \alpha c \beta & s \alpha s \beta s \gamma+c \alpha c \gamma & s \alpha s \beta c \gamma-c \alpha s \gamma \\
-s \beta & c \beta s \gamma & c \beta c \gamma
\end{array}\right]
\end{aligned}
$$




$$
\begin{gathered}
{ }_{C}^{B} R_{r p y}(10,37,75)=\left[\begin{array}{ccc}
\cos 75 \cos 37 & \cos 75 \sin 37 \sin 10-\sin 75 \cos 10 & \cos 75 \sin 37 \cos 10+\sin 75 \sin 10 \\
\sin 75 \cos 37 & \sin 75 \sin 37 \sin 10+\cos 75 \cos 10 & \sin 75 \sin 37 \cos 10-\cos 75 \sin 10 \\
-\sin 37 & \cos 37 \sin 10 & \cos 37 \cos 10
\end{array}\right] \\
{ }_{C}^{B} R_{r p y}(10,37,75)=\left[\begin{array}{ccc}
0.2067 & -0.922 & 0.3192 \\
0.77 & 0.353 & 0.5244 \\
-0.6 & 0.138 & 0.785
\end{array}\right]
\end{gathered}
$$$$
{ }_{B}^{A} R_{r p y}(45,15,30)=\left[\begin{array}{ccc}
\cos 30 \cos 15 & \cos 30 \sin 15 \sin 45-\sin 30 \cos 45 & \cos 30 \sin 15 \cos 45+\sin 30 \sin 45 \\
\sin 30 \cos 15 & \sin 30 \sin 15 \sin 45+\cos 30 \cos 45 & \sin 30 \sin 15 \cos 45-\cos 30 \sin 45 \\
-\sin 15 & \cos 15 \sin 45 & \cos 15 \cos 45
\end{array}\right]
$$$$
{ }_{B}^{A} R_{r p y}(45,15,30)=\left[\begin{array}{ccc}
0.836 & -0.1951 & 0.5119 \\
0.482 & 0.7036 & -0.52 \\
-0.258 & 0.6829 & 0.682
\end{array}\right]
$$$$
{ }^{B} P_{\text {CORG }}=\left[\begin{array}{l}
4 \\
7 \\
8
\end{array}\right] \text { and } \quad{ }^{A} P_{B O R G}=\left[\begin{array}{c}
2 \\
12 \\
-3
\end{array}\right] \text { and } \quad{ }^{C} P=\left[\begin{array}{c}
3 \\
4 \\
12
\end{array}\right]
$$$$
{ }_{B}^{A} R_{C}^{* B} R=\left[\begin{array}{ccc}
0.836 & -0.1951 & 0.5119 \\
0.482 & 0.7036 & -0.52 \\
-0.258 & 0.6829 & 0.682
\end{array}\right]\left[\begin{array}{ccc}
0.2067 & -0.922 & 0.3192 \\
0.77 & 0.353 & 0.5244 \\
-0.6 & 0.138 & 0.785
\end{array}\right]
$$$$
{ }_{B}^{A} R_{C}^{*} R=\left[\begin{array}{ccc}
-0.2863 & -0.7632 & 0.5563 \\
0.947 & -0.2642 & 0.1072 \\
0.065 & 0.5564 & 0.8061
\end{array}\right]
$$$$
{ }_{B}^{A} R_{C}^{* B} R={ }_{C}^{A} T=\left[\begin{array}{cccc}
-0.28 & -0.76 & 0.55 & 8.07 \\
0.94 & -0.26 & 0.1 & 14.6 \\
0.065 & 0.556 & 0.8 & 6.2 \\
0 & 0 & 0 & 1
\end{array}\right]
$$$$
{ }^{A} P={ }_{C}^{A} T^{C} P=\left[\begin{array}{cccc}
-0.28 & -0.76 & 0.55 & 8.07 \\
0.94 & -0.26 & 0.1 & 14.6 \\
0.065 & 0.556 & 0.8 & 6.2 \\
0 & 0 & 0 & 1
\end{array}\right]\left[\begin{array}{c}
3 \\
4 \\
12 \\
1
\end{array}\right]=\left[\begin{array}{c}
10.79 \\
17.58 \\
18.195 \\
1
\end{array}\right]
$$$$
{ }^{A} P=\left[\begin{array}{c}
10.79 \\
17.58 \\
18.195
\end{array}\right]
$$ 
As shown in figure (9) it is needed to reach point $\mathrm{P}$ which lies in frame $\{\mathrm{C}\}$ starting from the origin $\mathrm{O}$ in frame $\{\mathrm{A}\}$ passing through an intermediate frame $\{\mathrm{B}\}$ and to repeat that reaching many times to perform a specified task, so the researcher used three methods, they are:

a) Self coordinates feeding.

b) Manual alignment.

c) By using the suggested method.

At each method the manipulator returned to the same point $\mathrm{P}$ six times and the coordinates of the final point was measured to compare the obtained results with the standard one.

Figure (10) shows the obtained final point that reached by the end effecter as the manipulator feeding itself by the needed coordinates. Figure (11) indicates the second case which is called manual alignment, the gained points are best than those of the above case where the points are closer to the origin point. Figure (12) gives a clear idea about the obtained positions distribution around the origin point $\mathrm{O}$, they are very close to the origin and these results are best than the other previous two case.

\section{CONCLUSIONS \& RECOMMENDATIONS}

The conclusions that can be draw from this work are largely depend on the type of the manipulator considered, where it is so important to use new manipulator to avoid many accumulated errors. We can conclude that the obtained results by using the suggested method is very close to our target even when that test repeated many times and this depends on the high accuracy in using matrix compound transformation procedure which eliminates all kinds of an expected associated errors also it gives precise coordinates in terms of three dimensional recognition. Almost manipulators in our factories works under different working conditions such as variance of temperature, environment noise so we need to recalibrate the manipulator periodically to reduce the deviation in the base and end effecter coordinates but when using the above technique the manipulator will reach its goal in more precision and that very clear in the test of repeatability, so the researcher recommended to update all manipulator software according to the conducted research. Furthermore there is a way to use the matrix compound transformation method for different manipulators which means those 
manipulators with more than three joints and three links to extend the transformation to infinite number of joints and links with an accurate repeatable results.

\section{ACKNOWLEDGMENT}

The present work was undertaken under the support of the State Company for Mechanical Industries (SCMI), funded by Foundation of Technical Education (FTE). The work has been carried out at the factories of the SCMI. The author acknowledges the constant support encouragement of his colleagues at the FTE.

\section{NOMENCLATURE}

${ }^{A} P$

$P_{X}, P_{Y}, P_{Z} \quad$ Cartesian coordinates of point $\mathrm{P}$

$\{A\}, \quad\{B\}$,

$\{\mathrm{C}\}$

Frames

$X_{A}, Y_{A}, Z_{A} \quad$ Principal coordinates of any frame

$X_{B}, Y_{B}, Z_{B}$

$\{A\}$ or $\{B\}$

$r_{i j}$

${ }_{B}^{A} R \quad$ Rotation matrix

${ }_{B}^{A} R^{-1} \quad$ Inverse of a rotation matrix

${ }_{B}^{A} R^{T} \quad$ Transpose of a rotation matrix

$I_{3}$

${ }^{A} P_{B O R G}$

${ }_{B}^{A} T,{ }_{C}^{B} T,{ }_{C}^{A} T$
[3*3] Identity matrix

Vector locates the origin of $\{B\}$

Transformation matrix
SCMI

FTE $\begin{array}{ll}R_{X}(\gamma) & \text { Rotation matrix about } \\ & \mathrm{X}\end{array}$

$R_{Y}(\beta)$

$R_{Z}(\alpha)$

$\alpha, \beta, \gamma$

$c \alpha, c \beta, c \gamma \quad \cos (\alpha), \cos (\beta), \cos (\gamma)$

$s \alpha, s \beta, s \gamma \quad \sin (\alpha), \sin (\beta), \sin (\gamma)$
State Company for

Mechanical Industries

Foundation of

Technical Education

Rotation matrix about Y

Rotation matrix about Z

Angles of rotation 


\section{REFERENCES:}

1. Craig, John J., 1995 "Introduction to Robotics: Mechanics and Control” 2 ${ }^{\text {nd }}$. Ed., by: Addison Wesley Publishing Company.

2. Osita D. I. Nwokah, Yildirim Hurmuzlu, 2002 "The Mechanical Systems Design Handbook" by: CRC Press.

3. B. Z. Sandler, 1999 "Robotics Designing and Mechanisms for Automated Machinery" $2^{\text {nd }}$. Ed. by: Academic Press.

4. R. P. Paul, 1981, "Robot Manipulators” MIT Press.

5. Stephen Derby, 2005 "Design of Automatic Machinery" by: Marcel Dekker, New York.

6. Y. Koren, 1985, "Robotics for Engineers” by: McGraw Hill.

7. V. Hunt, 1983, "Industrial Robotics Handbook” by: Industrial Press.

8. G.M. Acaccia, P.C. Cagetti, M. Callegari, R.C. Michelini, R.M. Molfino. 1994. "Modeling the Impact Dynamics of Robotic Manipulators", Preprints 4th IFAC Symp. on Robot Control: SY.RO.CO. '94, Capri, Sept. 19-21. 559-564.

9. R.M. DeSantis. 1996, "Motion and Force Control of Robotic Manipulators" ASME J. Dynamic Systems, Measurement and Control, 118(2): 386-389.

10. Cornelius Leondes, 2001 “Artificial Intelligence and Robotics in Manufacturing” Vol. VII. By: CRC press. Chapter 10.

11. Neil Sclater, Nicholas P. Chironis, 2001 "Mechanisms and Mechanical Devices Sourcebook" $3^{\text {rd }}$. Ed. By: McGraw Hill.

12. Y. Fang, Z. Huang. 1997,'Kinematics of a Three-Degrees-of-Freedom in- Parallel Actuated Manipulator Mechanism, Mechanism and Machine Design” 32(7): 789-796.

13. Tom M. Apostol 1969, “Calculus” $2^{\text {nd }}$. Ed., Vol. I\&II, by: John Wiley \& Sons.

14. John Bird, 2003 "Engineering Mathematics" $4^{\text {th }} . E d .$, by: Newnes.

15. David S. Watkins, 2002 "Fundamental of Matrix Computations" 2nd. By: John Wiley \& Sons

16. Carl D. Meyer, 2000 "Matrix Analysis and Applied Linear Algebra” by: SIAM.

17. Gordon M. Mair, 1988 “Industrial Robotics”, by: Prentice Hall, UK.

18. Werner G. Holzbock, 1986 "Robotic Technology Principles and Practice" by: Van Nostrand Reinhold, New York. 


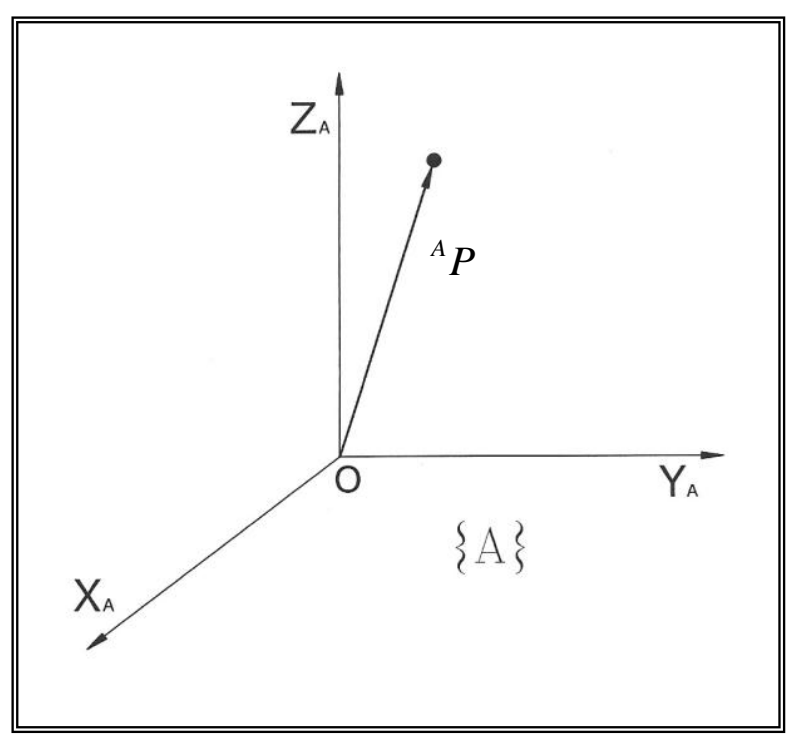

Figure (1): Vector relative to frame example.

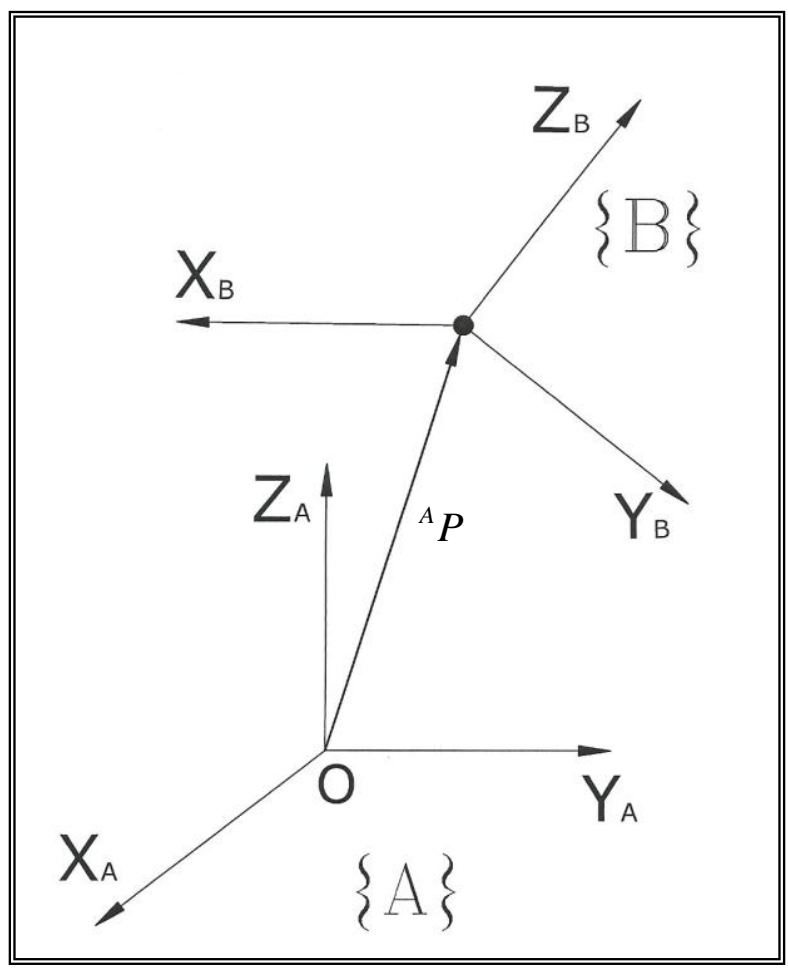

Figure (2): Locating an object in position and orientation. 


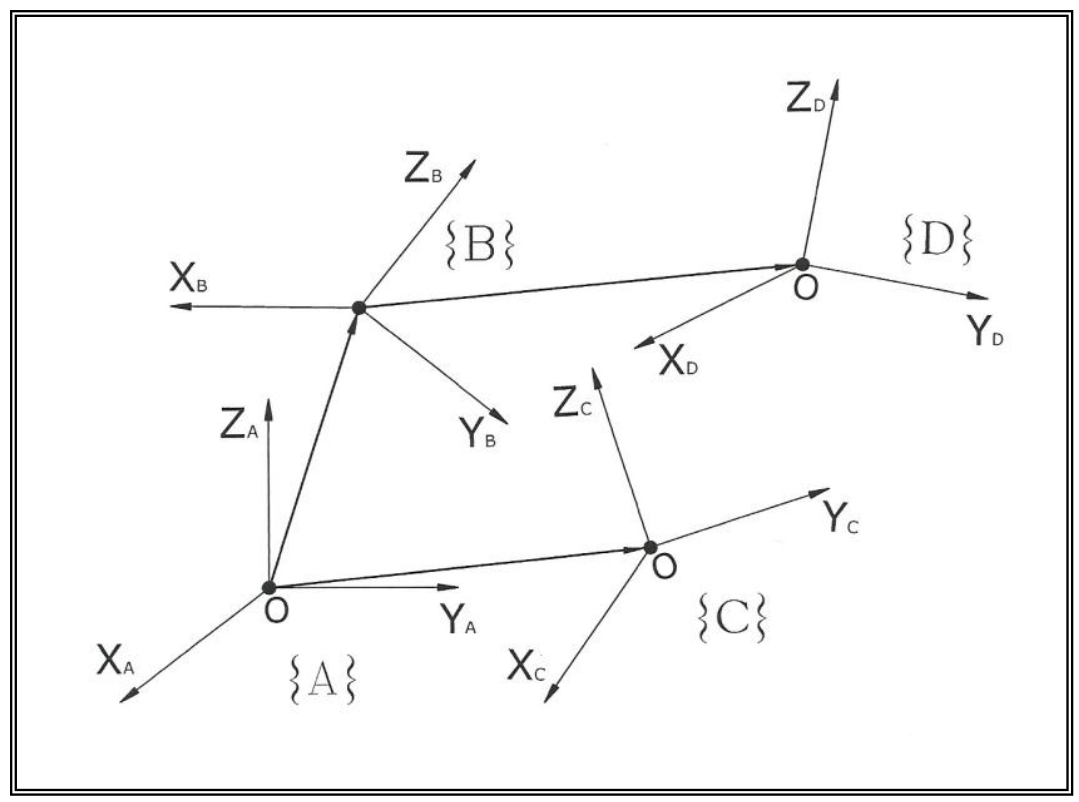

Figure (3): Examples of several frames

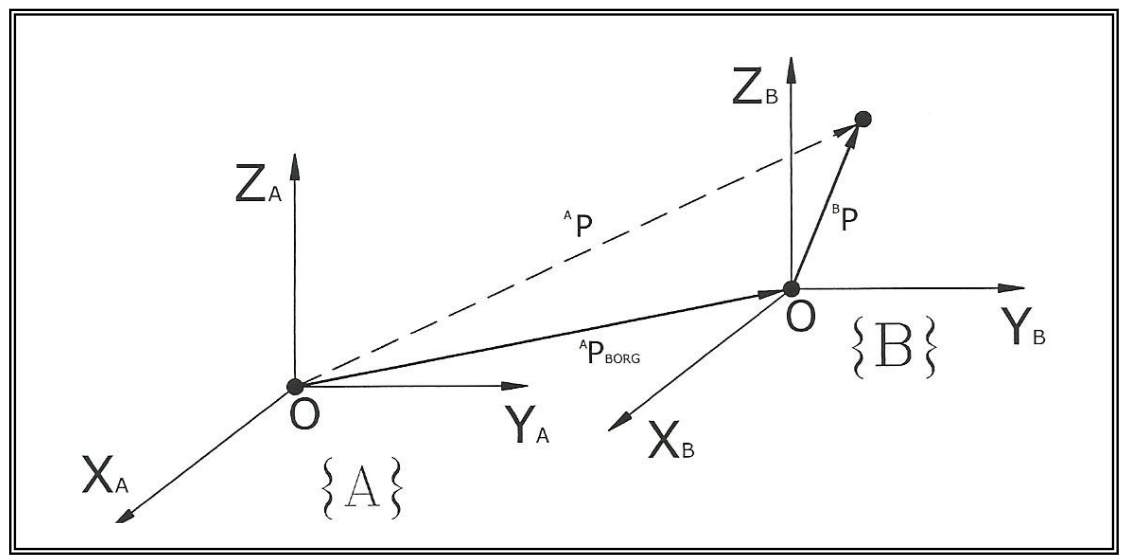

Figure (4): Translation mapping.

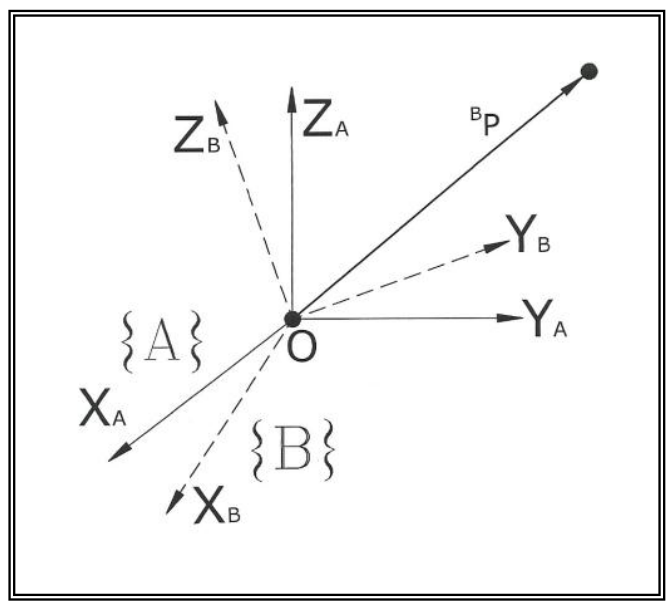

Figure (5): Rotating the description of a vector. 


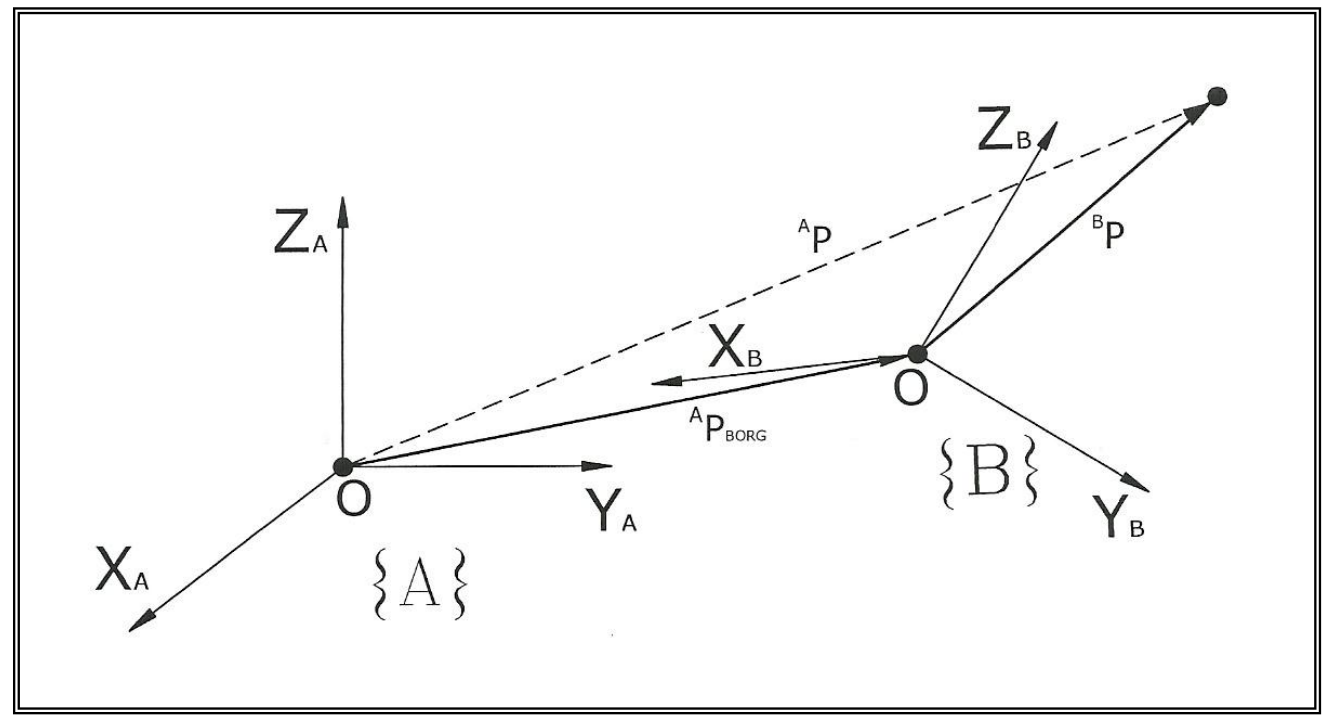

Figure (6): General transform of a vector.

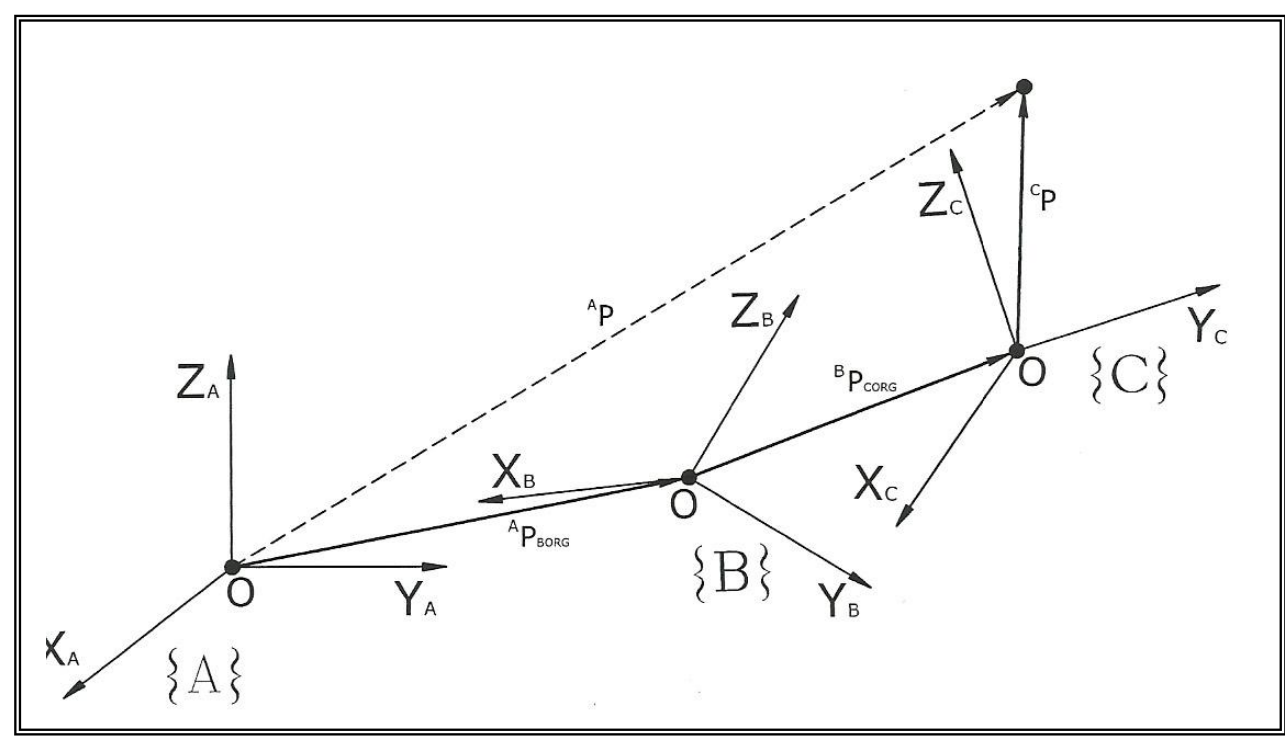

Figure (7): Compound frames each is known relative to previous.

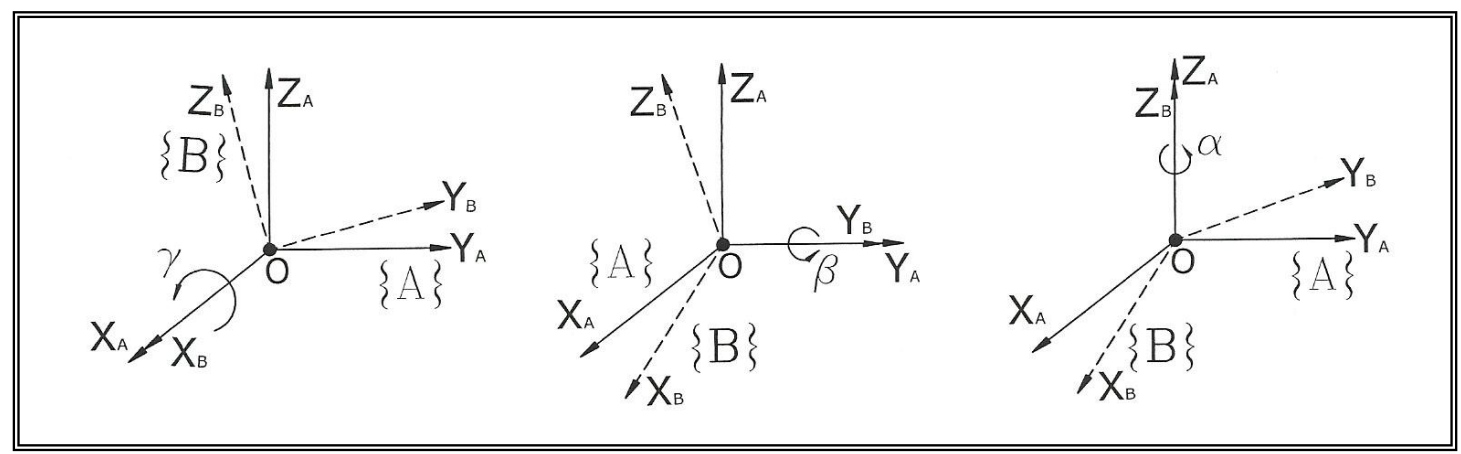

Figure (8): X-Y-Z fixed angles. Rotation are performed in the order $R_{X}(\gamma), R_{Y}(\beta)$, $R_{Z}(\alpha)$. 


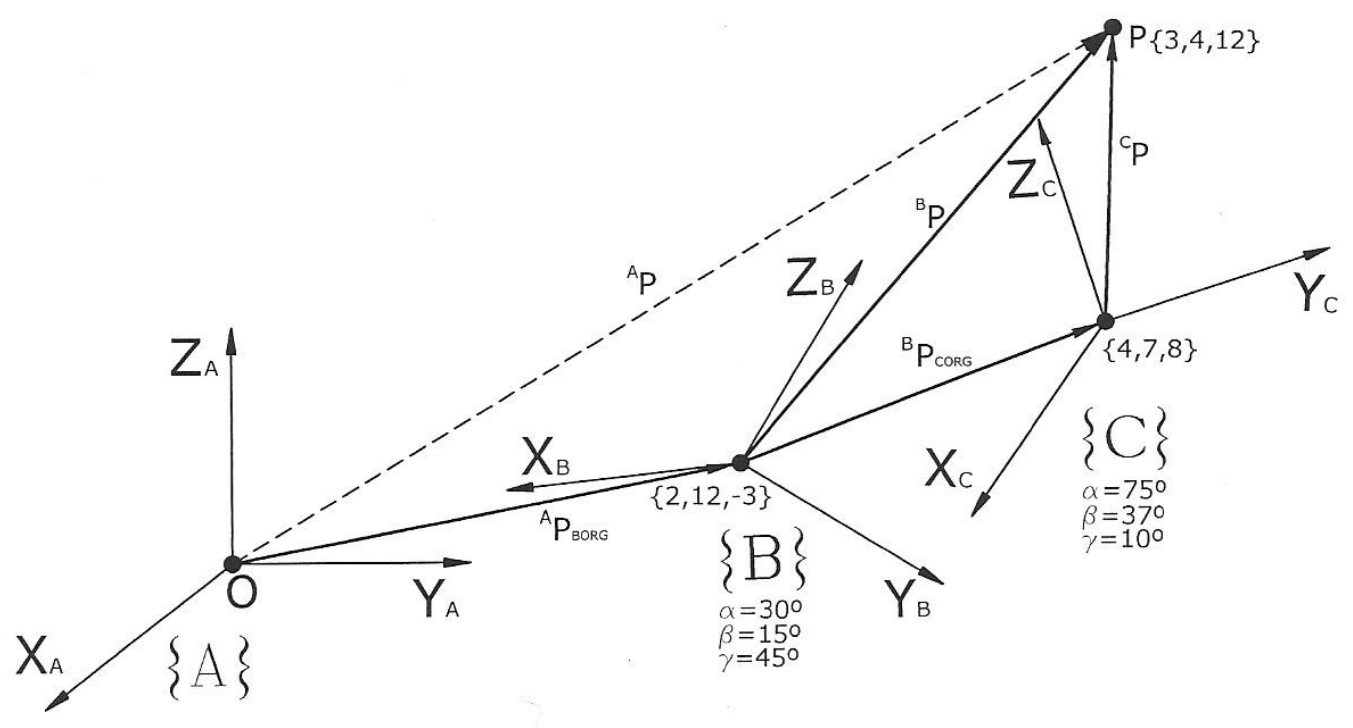

Figure (9): Three frames with compound transformation.

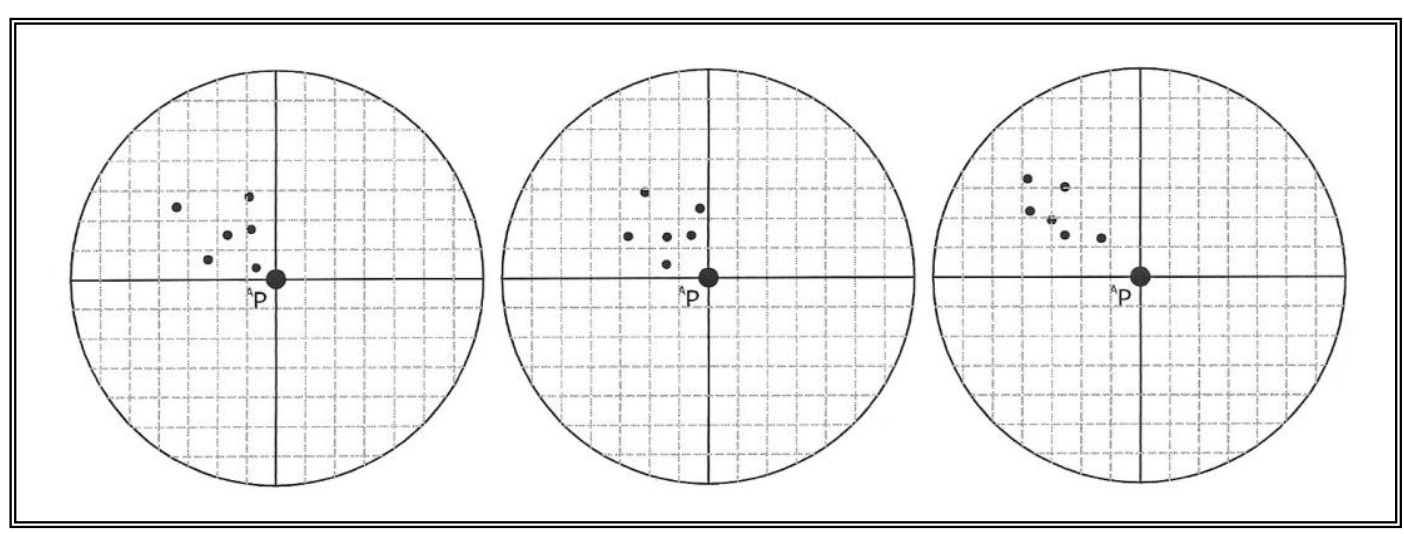

Figure (10): Self coordinates feeding results.

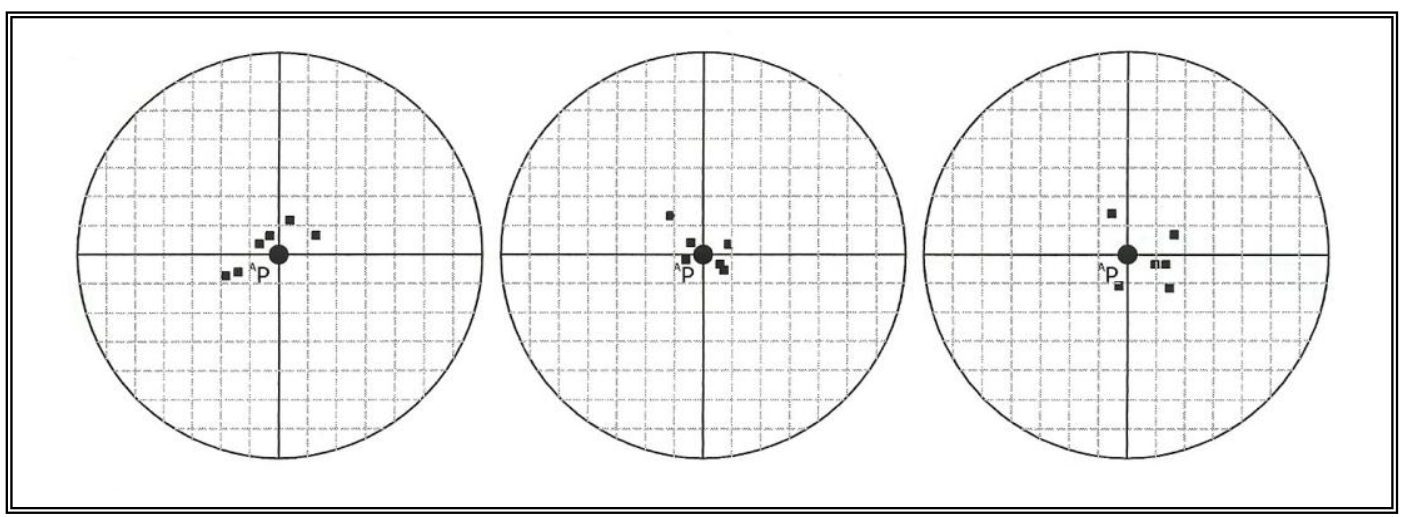

Figure (11): Manual alignment results. 


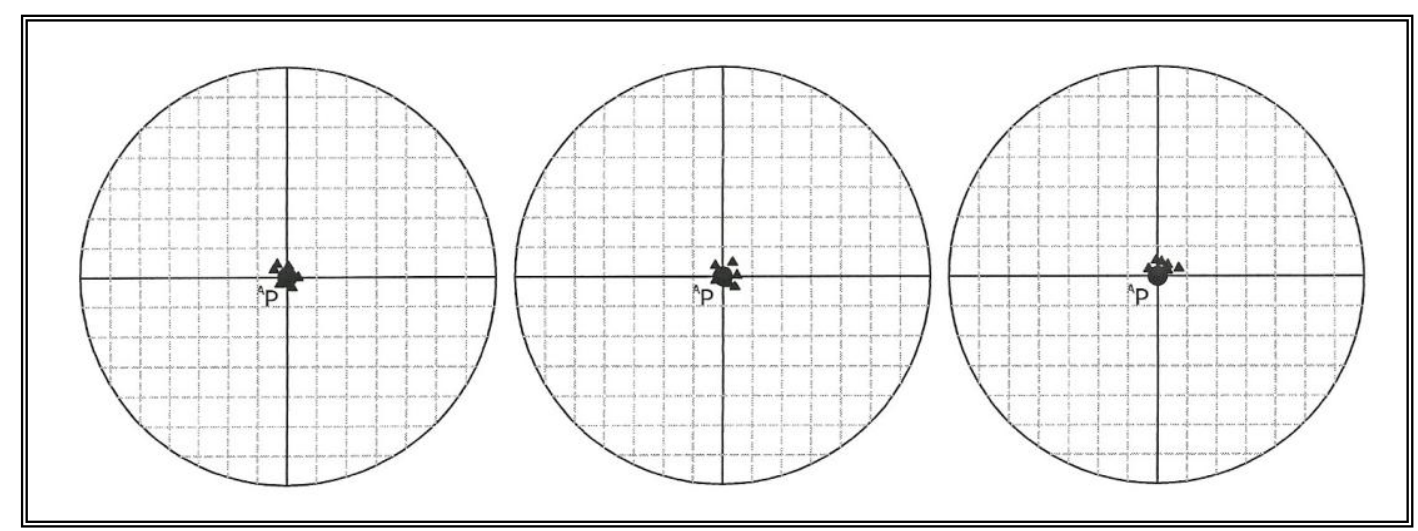

Figure (12): The suggested method results. 


\title{
قياس الاحداثيات الايكارتية ثلاثية الابعاد لنهاية ذراع رويوت بالاعتماد على هياكل مركبة
}

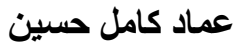 \\ مدرس \\ الكلية التقنية/ المسيب
}

في هذا البحث تم القيام بدراسـة نظريـة وعمليـة لقياس الاحداثيات الديكارتيـة ثلاثيـة الابعـاد لنهايـة ذراع روبوت بالاعتماد على ثلاثة هياكل او نظام محاور ثلاثثية متعامدة مختلفة كل منها معرف نسبة الى الى الاخر .

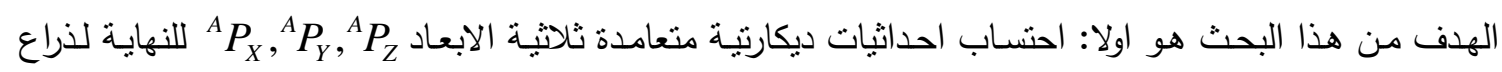

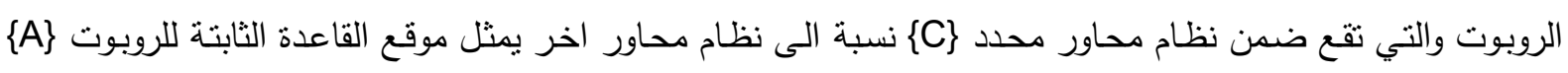

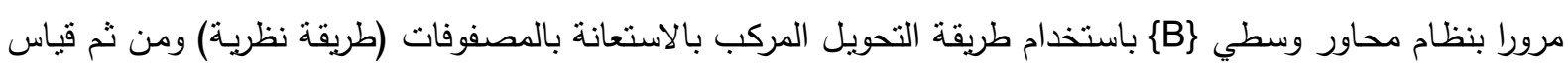
نفس الاحداثيات لنهاية الذراع وباستخدام طريقيتين تقليديتين هما: O

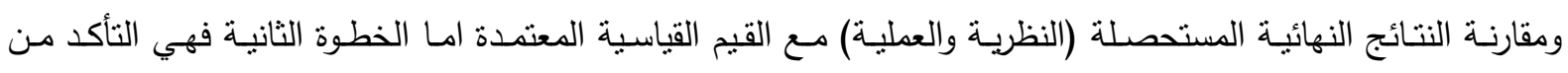
الرجوعية (Repeatability) بالنسبة لكل الطرق الثلاثة المذكورة اعلاه وذلك باعادة عملية القياس للاحداثيات النهائية بتكرار 18 مرة ولكل طريقة قياس. أظهرت النتائج النهائية ان طريقة التحويل المركب بالاستعانة بالمصفوفات تعطي نتائج رجوعية اكثر دقة من الطريقتين الاخربين. 